\title{
Bensa Alban et Isabelle Leblic (sous la direction de), En pays kanak
}

2000, Mission du Patrimoine ethnologique, Collection Ethnologie de la France, cahier 14, éditions de la Maison des Sciences de l'Homme, Paris, $368 \mathrm{p}$.

\section{Hamid Mokaddem}

\section{(2) OpenEdition}

\section{Journals}

Édition électronique

URL : http://journals.openedition.org/jso/1631

DOI : 10.4000/jso.1631

ISSN : 1760-7256

Éditeur

Société des océanistes

\section{Édition imprimée}

Date de publication : 1 décembre 2001

Pagination : 224-227

ISSN : 0300-953x

\section{Référence électronique}

Hamid Mokaddem, «Bensa Alban et Isabelle Leblic (sous la direction de), En pays kanak », Journal de la Société des Océanistes [En ligne], 113 | Année 2001-2, mis en ligne le 27 mai 2008, consulté le 24 septembre 2020. URL : http://journals.openedition.org/jso/1631; DOI : https://doi.org/10.4000/jso. 1631

(c) Tous droits réservés 
sociales à chaque fois spécifiques et un ordre cosmique qui, en dernière instance, en informe la cohérence. Autre trait fondamental, le cumul possible des malheurs chez un même individu: "dans les atteintes atypiques », nous dit Christine Salomon, «[...] lorsqu'une causalité a priori n'est pas déterminable selon le code étiologique local, intervient la notion de maladies superposées et surajoutées les unes aux autres ». Cette sorte de diagnostic offre l'indéniable avantage d'éluder « la difficile question du diagnostic différentiel qui hante les consciences médicales occidentales" ainsi que d'éviter ultérieurement que «l'échec du traitement n'invalide complètement le diagnostic et la compétence du praticien » et, au delà, la puissance reconnue aux ancêtres dont le thérapeute n'est que le médiateur. "S'il y a échec, souligne l'auteur, c'est que le pouvoir de la voyance n'était pas assez fort pour dénombrer toutes les maladies ; ce que le premier spécialiste du diagnostic étiologique n'a pu voir jusqu'au bout, peut-être alors un second pourrat-il le détecter totalement » (p. 101).

Le rôle des thérapeutes et les modes de traitement sont décrits et analysés de manière approfondie dans la troisième partie du livre. "Voir la maladie" est l'affaire de deux types d'intervenants. Ceux dont la clairvoyance est un legs ancestral personnel, fruit d'un rêve ou d'une vision - leur consultation suppose un minimum des gestes rituels - , et ceux dont les pouvoirs passent une transmission initiatique et impliquent techniques divinatoires de consultation des ancêtres, invocations et offrandes. Toutefois, la démarche est grosso modo la même et vise à atteindre à une identification la plus complète possible des racines sociales de la maladie. Si la cause en est une faute commise, le voyant fait des propositions concrètes pour sa résolution (réparation de l'infraction par un don cérémoniel et indications thérapeutiques). S'il s'agit d'une agression par sorcellerie, il joint au traitement des mesures de protection rituelle plus draconiennes, voire suggère d'entrer en "guerre " avec l'agresseur. La cure se transforme alors en une véritable combat à mort dont les résultats ne sont jamais acquis.

La plupart des questions touchant aux modalités thérapeutiques traditionnelles sont traitées en détail par Christine Salomon : le travail crucial du guérisseur dont la parole (le souffle) médiatise les pouvoirs thérapeutiques de l'ancêtre vers la plante ou l'association de plantes données au patient, durée du traitement, horaires de cure, fonction des interdits alimentaires, sexuels et/ou touchant la toilette quotidienne (totalement ou partiellement partagés par le thérapeute et son patient). Mais j'avoue que c'est la description du rôle des éléments (eau, terre, feu et vent) qui m'a le plus intéressé et, même, impressionné dans cette partie. Elle permet d'appréhender la nature réelle de l'action entreprise par le guérisseur qui n'est pas seulement d'interrompre la maladie, mais bien plus profondément de restaurer l'équilibre des composantes internes de la personne malade en s'aidant des éléments fondamentaux du cosmos. Lieu d'échanges permanents " entre le monde interne et le monde externe " et « entre les ancêtres et les vivants » pour reprendre les termes déjà cités de Christine Salomon, la personne guérit grâce à une intervention rituelle qui rétablit en elle le même ordre vital qui gouverne aussi son environnement.

La conclusion du livre est principalement consacrée à la rencontre entre médecines kanak et européenne et à l'avenir des savoirs et pouvoirs thérapeutiques traditionnels dans un monde en plein changement. La réponse actuelle des communautés kanak consiste en un pluralisme des plus réalistes. Qu'en sera-t-il demain ? La réponse appartient moins aux anthropologues qui font rarement de bons devins qu'aux soignants de Nouvelle-Calédonie. La médecine kanak a su «jusqu'à présent éviter les ruptures brutales que pourrait entraîner le choix exclusif d'un système, protéger l'essentiel de ses conceptions en y intégrant pragmatiquement des éléments nouveaux ». Il revient, selon moi, à la médecine occidentale, dite savante, et aux politiques locales de santé de faire maintenant à leur tour une place à la médecine kanak.

Pour conclure ce compte-rendu bien infidèle, je voudrais insister sur l'importance du travail de Christine Salomon et dire combien il contribue à renouveler en profondeur la vision donnée jusqu'alors de cette partie du monde océanien qu'est la Nouvelle-Calédonie. En effet, ce livre ne se contente pas de mettre en évidence les rapports qui existent ici entre conception de la maladie, et plus largement du malheur, et cosmologie, ou d'insister sur la part irréductible d'indétermination et d'incertitude qui frappe toute interprétation de l'irruption du mal dans une organisation sociale où les relations priment sur les personnes, il fait saillir le lien quasiment organique que le corps humain, sa santé et ses affections entretiennent avec le fonctionnement et le développement du « corps social ». Au delà des faits de maladie et des savoirs et savoir-faire thérapeutiques kanak, c'est dans la compréhension de ces échanges, de cette logique de la communication que, je crois, réside l'apport majeur des recherches de Christine Salomon. Et la leçon dépasse assurément de beaucoup le simple intérêt ethnographique.

\section{Patrice GodIN}

chargé du patrimoine à la Province Nord (Nouvelle-Calédonie)

BENSA Alban et Isabelle LEBLIC (sous la direction de), 2000. En pays kanak, Mission du Patrimoine ethnologique, Collection Ethnologie de la France, cahier 14, éditions de la Maison des Sciences de l'Homme, Paris, 368p.

\section{Un outil d'analyse?}

Il est impossible matériellement de rédiger un compte rendu exhaustif d'une œuvre collectant des contributions aussi diversifiées. Les seize textes, sans compter les avant-propos, traversent cinq disciplines (ethnologie, sociologie, archéologie, linguistique et histoire), traitent de champs et de découpes d'objets hétérogènes, allant des itinéraires des clans en conflit 
pendant la période des XVIII ${ }^{\mathrm{e}}$ et $\mathrm{XIX}^{\mathrm{e}}$ siècles aux migrations kanak vers Nouméa. Projet ambitieux qui couvre la période antécoloniale des sociétés kanak à leur transformation historique par le « choc colonial». Ce programme s'ouvre vers une perspective pragmatique : comprendre et faire comprendre les sociétés kanak dans leur devenir. Ces jeux complexes de transformations touchent aussi bien le rapport comportemental à la santé, les règles de grammaire ou les règles de transfert des enfants. La méthodologie ne peut plus être celle d'une anthropologie structurale mais celle qui s'efforce de resituer les modifications structurales dans des contextualisations historiques ou dans des cadrages d'échelles plus fines que les échelles macroscopiques qu'on trouve dans la batterie standard de l'opposition tradition/modernité, ou de la naissance de la tradition, pour ne pas mentionner les classiques théories de l'impact de l'Occidentalisation sur les microsociétés kanak. Néanmoins, pour construire une intelligibilité d'un ensemble cohérent, les éditeurs Alban Bensa et Isabelle Leblic ont décidé de répartir en trois rubriques ces communications : Pratiques et règles, Retour sur le choc colonial, Initiatives nouvelles. Cette répartition tripartite se fonde sur une supposition implicite qui est que les sociétés kanak ne sont pas figées. Pour reprendre un mot célèbre de JeanMarie Tjibaou, les sociétés kanak se transforment en reformulant les pratiques et les règles dans des initiatives nouvelles. Vue sous cet angle, il est clair qu'une note de lecture ne peut se substituer à l'effort d'analyse du lecteur et encore moins imposer un ordre de lecture.

Pour éviter le style acerbe de la polémique gratuite sur un livre qui mérite un traitement digne nous commencerons par nous débarrasser de deux remarques futiles.

Ces textes résultent d'enquêtes de terrain ciblées, de thèses universitaires de jeunes chercheurs ou de projets précis. Tous ces travaux ont été financés par l'État pendant la période des accords de Matignon. La note 1 de la page 2 indique que ces contributions résultent de travaux de recherche financés par le programme ESK (étude des sociétés kanak). Alban Bensa et Isabelle Leblic ont retenu les textes les plus lisibles et pertinents dans l'objectif et l'intention que ce livre constitue un outil de pensée, d'action et de dialogue (p. 6). Propos repris de Jean-Marie Tjibaou. Un outil n'a de valeur que dans l'usage qui en est fait par les concernés. Il faut souhaiter du courage aux Kanaks parce que, si belles soient ces contributions, elles ne sont pas pour autant aisées de lecture. Le propos de Jean-Marie Tjibaou, sauf erreur de notre part, se trouve dans l'entretien consacré à Jacques Violette, en 1987, reproduit dans le numéro de Bwenando consacré au message de JeanMarie. Il dit être parti en 1968 en France non pas pour étudier mais pour se forger des outils d'analyse. Ce qui signifie que ces outils, il se les est forgé lui-même et que cet outillage n'a strictement aucun rapport avec la rhétorique mystico-coutumière d'Octave Togna qui fait allusion aux «bouts de paroles» des découpes disciplinaires par rapport à la parole INFINIE kanak. Octave Togna veut sans doute dire parole indéfinie ou qui n'en finit plus, qui ne peut pas être délimitée dans la découpe scientifique d'un objet. Cette parole sacrée, qui se ressource à la niche des ancêtres, féconde les discours savants :

« L'ethnologie, la linguistique, l'histoire, l'archéologie ne sont que des « bouts » de parole de notre société. Elles puisent leur fondement dans les techniques d'analyse. Mais pour être consistantes, elles ne peuvent prendre source que dans cette parole qui n'a pas de fin, car la parole est INFINIE » (avant-propos, p. ix).

On pourrait objecter au propos d'Octave Togna qu'il n'y a pas qu'une société kanak. La première personne du pluriel («notre société») fait jouer une identification de groupe n'ayant de sens que dans le champ idéologique mais qui perd de sa pertinence dans le champ de l'investigation du réel. Les microsociétés kanak de la Grande Terre ont-elle une homogénéité structurale avec celles des Îles Loyauté ?

Il est vrai que l'avant-propos d'Octave Togna finit sur une remarque judicieuse, à savoir que «cette parole et ce regard sont redoutables » mais qu'ils " nous aident à mieux comprendre les sociétés dans lesquelles nous vivons » (ibid. $\mathrm{p}$. ix et $\mathrm{x}$ ).

Si la compréhension pouvait contribuer à modifier les comportements dans le rapport à soi et aux autres, l'anthropologie vaudrait une heure de peine et mériterait bien ce titre élogieux de connaissance de soi à travers la connaissance de l'autre. Ce livre aurait réussi du même coup son objectif qui est de « se réapproprier ces analyses pour en faire des 'outils' de pensée, d'action et de dialogue » (avant-propos d'A. Bensa et I. Leblic, p. 6). Ces remarques peuvent nous faire comprendre que le côté pragmatique et fonctionnel n'est pas dissociable du côté sacré et spirituel en Océanie.

Passons au contenu et proposons une lecture diagonale pour retenir des lignes de force de ce livre. Celui-ci s'ouvre sur une contribution ethno-historique d'Alban Bensa sur la variation du modèle de « chef kanak ». Les modèles de la chefferie proposées par les récits européens et les explications ethnologiques recoupent-ils les pratiques réelles des clans? $\mathrm{Ce}$ concept de « chef kanak », comme celui de « clan », de " coutume » ou de " boucan », est devenu un terme écran qui cultive des ambiguïtés. Il participe, au même titre que celui de «tribu », d'une catégorie fine de la construction coloniale. Pour retracer la généalogie de ce concept, Alban Bensa, qui travaille et collecte des récits depuis 1973 dans le centre nord-ouest de la Nouvelle-Calédonie, a recours à une écriture de l'histoire ethnographique de Koné. Dans un autre travail collectif, il s'est expliqué sur cette démarche qui fait appel à un jeu d'échelles. À l'échelle macroscopique de l'histoire européenne, il s'agit de compléter par la perception plus fine de l'échelle microhistorique de l'ethnographie, à savoir de l'histoire des clans recueillis dans les récits. Les historiens kanak comme Antoine Goromido et Emmanuel Neaouna relatent des itinéraires de clans. La superposition de ces récits avec les archives écrites comme les points de vue des santaliers, 
premiers européens à être en contact avec les Kanaks, s'avère nécessaire pour la compréhension des confusions sur les définitions des chefferies. Ces dernières sont à resituer dans un contexte historique déterminé et des jeux subtils et compliqués entre la pacification blanche et les conflits internes entre les chefs kanak. Retracer l'histoire de ce modèle de la chefferie suppose que l'anthropologue doit s'efforcer de repérer les topiques où « les archives et la mémoire kanak se superposent [...] presque exactement » (p. 33).

L'analyse du système politique kanak élucide ces jeux de transformation historique de la chefferie. La description idéalisée du chef renvoie à la période historique de la paix blanche (Maurice Leenhardt). Le don de la chefferie à un étranger (Jean Guiart et Alban Bensa lui-même) renvoie à des calculs d'alliances entre clans pour apaiser des rivalités néfastes dans un contexte historique précis. Que faut-il déduire de cette disparité des figures politiques de la chefferie ? Alban Bensa démontre que ni le recours à l'analyse formelle, qui cherche une essence ou un type de la chefferie avec des variations successives dans une série, ni l'appui culturaliste sur des schèmes anhistoriques d'une chefferie atemporelle, ne sont pertinents pour comprendre cette disparité.

«À l'inverse, l'histoire politique de la moyenne Koné montre que ces comportements, quand on les rapporte à la chronologie des faits, se comprennent comme des solutions temporaires à des problèmes contingents. En tant que telles, au cours d'une période donnée, les décisions politiques kanak effectives révèlent des complexités et des contradictions essentielles irréductibles à des figures statiques » (p. 41).

On pourrait chercher un recoupement avec la contribution des archéologues ayant travaillé dans la même région Tiwaka-Koné (pp.171 et $s q$.). En vain. Les tertres, traces matérialisées des sites des itinéraires des clans, sur lesquels enquêtent les archéologues, ne recouvrent pas la même épaisseur historique. L'ethnologie « étudie toujours un héritage historique récent 》 (p. 42).

La communication d'Isabelle Leblic porte sur une lecture plus fine des pratiques courantes en pays kanak de l'adoption des enfants. L'hypothèse d'AndréGeorges Haudricourt sur la xénophilie des sociétés kanak qui cultivent des boutures exogènes qui est à mettre en parallèle avec la pratique fréquente des « échanges d'enfants» n'est pas rejetée. Mais elle nécessite de « resserrer l'analyse » de ces circulations de personnes « autour de situations précises » (p. 51). Le terrain des enquêtes sur la parenté est aussi le pays paicî. Recadré dans une région plus précise, celle de Ponerihouen, centre nord-est de la NouvelleCalédonie. Après une explication des échanges restreints autour des deux moitiés matrimoniales « Dui » et « Bai » et après avoir proposé une formalisation actuelle structurale des règles d'alliance à l'aide d'un logiciel conçu par l'économiste Jean Freyss, l'analyse discerne les différences subtiles entre l'adoption et le transfert d'enfants. Elle finit par interroger sur la motivation de ces pratiques en récusant la thèse de l'adop- tion comme compensation d'un vide dans la lignée matrilinéaire. Elle critique aussi la thèse soutenue par $\mathrm{S}$. Lallemand qui consiste à affirmer que les règles d'adoption modifieraient les règles de filiation et d'héritage. Les adoptions d'étrangers ou d'adultes sont plus souples et plastiques et beaucoup moins structurales. Il est à remarquer que les échanges de noms sont une pratique fréquente. Le petit chef de Iambé porte le nom du célèbre ethnologue Jean Guiart. Mais on pourrait peut-être reprocher à Isabelle Leblic de mettre sous silence la classification par les jeux de noms et surnoms qui sont une des facettes aussi de cette souplesse des jeux de transferts et des adoptions. Ainsi, la polémique autour du centre culturel Tjibaou inauguré le 5 mai 1998 à Nouméa provient du fait qu'une partie des clans de Hienghène n'ont pas accepté le transfert du nom du célèbre leader indépendantiste.

Ces deux contributions font partie de la première partie. Nous irons un peu plus vite et laisserons au lecteur la décision des choix de lecture. Toutes ces contributions de qualité mériteraient une lecture plus soutenue. Marie Pineau-Salaün, qui a consacré une thèse au sujet de l'école en Nouvelle-Calédonie, tend à démontrer dans Histoire et mémoire d'une institution coloniale, la scolarisation des Kanak au temps de l'indigénat que la réappropriation de l'école par les Kanaks ne peut se comprendre que si on couple à l'analyse sociologique de l'institution scolaire la dimension historique (l'archive) et la dimension ethnologique (le point de vue des acteurs sur leur trajectoire scolaire). Face à la myopie des analyses sociologiques, il s'agit de retracer l'histoire de l'école coloniale et d'entendre les récits des vieux Kanaks eux-mêmes. Ceci suppose un retour à la thèse classique de Maurice Halbwachs sur la mémoire collective. En fait, nous sommes encore dans la démarche inaugurée par Alban Bensa, à savoir faire recouper l'archive et le récit pour avoir une compréhension adéquate des pratiques kanak. Il s'agit aussi d'être à l'écoute de la parole des vieux. Ce que fait aussi en partie Eric Soriano dans Tisser des liens politiques, mobilisation électorale et vote mélanésien (1946-1958) (pp. 235-252).

Dans la troisième et dernière partie, Initiatives nouvelles, la contribution de Christine Salomon reprend une problématique anthropologique mise sur le devant de la scène par Pierre Bourdieu, à savoir celle de $l a$ domination masculine. (Dans cette partie, le livre se clôture sur la migration des gens de Houaïlou où Michel Naepels dément l'image idéalisée du séjour paisible des tribus). La contribution de Christine Salomon risque de heurter l'opinion et la sensibilité des hommes kanak. L'analyse reprend les données et acquis sur la catégorisation sexuée du monde, des pratiques, des plantes et des aliments que les langues ajië et paicî attestent dans leur vocabulaire et syntaxe. La dualité n'est pas une parité mais une disparité. Ainsi, les catégories lexicales de haut et de bas qui articulent la hiérarchie sociale se calquent sur l'opposition entre masculin/féminin. La hiérarchie se reproduit dans le rapport sexuel. Ce qui n'est pas sans rappeler les dernières innovations de l'archéologie de Michel Foucault dans son Histoire de la sexualité. 
«Un homme ne peut laisser une femme se mettre au-dessus de lui sous peine de voir son ventre se mettre alors à grossir comme dans une grossesse » (p. 315). Cette croyance n'est pas propre aux régions linguistiques dans lesquelles a enquêté Christine Salomon. Nous l'avons entendue un peu partout dans tous les pays kanak. Marcel Mauss dans Les techniques $d u$ corps esquissait déjà des pistes d'investigation sur les techniques de reproduction ${ }^{1}$. Dans la même observation ethnolinguistique, la pollution féminine, par les maladies sexuellement transmissibles, se dit en paicî wâtemû (case féminine) par opposition à la grande case masculine. Les pratiques des femmes kanak démontrent des positions moins figées vis-à-vis des règles traditionnelles de la domination masculine. $\mathrm{La}$ conclusion semble ouverte puisqu'elle finit sur une note positive : « les formes de vie des femmes, à l'heure actuelle, sont moins verrouillées qu'il n'y paraît de prime abord » (p. 336).

On laissera au lecteur la liberté de lecture. Nous retiendrons, à propos de ce livre collectif, l'innovation dans la pratique scientifique. Nous émettrons des réserves et des désaccords sur certaines enquêtes ou sur quelques points. Par exemple, la volonté, de la part de certains jeunes docteurs, d'avoir des partis pris de choix d'école. Ou d'être pris dans un formalisme et une logique scolastique qui consiste à poser d'entrée de jeu que la thèse avancée est nouvelle par rapport à des travaux précédents. Mais, comme disait Jacobson, la science est polémique. L'ouvrage suscite des réflexions et des prolongements dans l'enquête. En ce sens, on retrouve l'infinité de la parole qu'évoquait l'avantpropos d'Octave Togna.

\section{Hamid MoKadDEM}

IFMNC, Nouméa (Nouvelle-Calédonie)

'Uvea-Wallis : Une île pêchée par les dieux. Musée des Beaux-Arts de Chartres. Catalogue de l'exposition présentée en 2000 ; 160 p., lexique, bibliographie, 154 illustrations, 2 cartes.

Pour la première fois une exposition présentait en France ce Territoire français d'Outre-mer. Ce point remarquable sert en quelque sorte d'entrée en matière au catalogue, seul ouvrage à présenter ainsi le panorama actuel de cette île du Pacifique et son ancienne culture. Claude Stéfani, attaché de conservation au musée, et Hélène Guiot, archéologue, en sont les principaux organisateurs et rédacteurs : trois et onze articles respectivement. Ils ont su s'entourer de personnes très au fait des domaines qu'ils traitent et ceci donne richesse et vie à l'ensemble. Si la longueur et l'écriture des articles diffèrent, l'unité est donnée par un même souci de faire entrer pleinement le lecteur dans chacun des thèmes abordés. À tous, novice ou amateur éclairé, est offert ainsi la possiblilité d'aborder, avec sûreté et plaisir, des rivages lointains qu'il est rarement donné d'approcher.

L'avant-propos expose le contexte dans lequel les collections uvéennes se sont constituées. D'entrée de matière, l'ouvrage met en lumière la particularité de l'étude des collections océaniennes. À la rareté des objets collectés par des missionnaires — pour la plupart ici, des militaires et quelques administrateurs, dont le cas exceptionnel de L.-J. Bouge — s'ajoute, pour la Polynésie occidentale notamment, la difficulté à déterminer avec certitude la provenance des pièces. Wallis, souvent associée à Futuna, illustre la nécessaire prise de conscience de la circulation d'éléments culturels au sein de ce « parfait ensemble de zone d'échanges culturels intenses ». Les îles n'ont cessé de s'influencer depuis toujours, rappelle-t-on, d'où le besoin d'assouplir certaines classifications régionales. Ici sont habituellement définis trois ensembles culturels : Fidji, les îles Samoa (qui surtout influencèrent Futuna) et les Tonga (ayant pour leur part marqué 'Uvea, autrement dit Wallis).

De ces premières remarques découle la logique de l'organisation de l'exposition, et du catalogue, en trois parties. Le visiteur, et le lecteur, sont en premier point instruits du maillage de Terre et d'Histoire auquel l'île appartient, comme le rappel Marie-Claire BatailleBenguigui. Cette spécialiste de la culture polynésienne aborde le passé de la zone avant que Daniel Frimigacci et Frédéric Angleviel, chacun dans leurs domaines, nous introduisent à l'histoire et la culture même de l'île. C'est ainsi que nous est retracé ce que fut l'aventure humaine du peuplement, pour les pécheurscéramistes Lapita, au cœur de ce "vaste et premier complexe micro-insulaire polynésien ». Frédéric Dupuy et Hélène Guiot donnent les nécessaires repères de géographie humaine. Le tout introduit au descriptif des pièces en provenance d'îles alentour: Futuna, Tonga, Samoa, Fidji, Tokelau, Niue, Rotuma.

Sur les $96 \mathrm{~km}^{2}$ de terres volcaniques qui la constitue, 'Uvéa l'ancienne, rebaptisée Wallis, dit l'histoire du travail des hommes ; c'est le thème de la seconde partie. Cette odyssée humaine a pour commencement l'implantation d'une population céramiste, notamment à 'Utuleve. Ce site du district de Mu'a donnera son nom à trois séquences à céramique de la préhistoire uvéenne jalonnée de périodes successives, étudiées et présentées par l'archéologue Daniel Frimigacci. Celle-ci débute, entre 1370 et 1320 avant J.-C., par un habitat, peut-être sur pilotis, qui se situe face à des passes du récif, sur des plages de galets et dunes, à proximité de zones marécageuses. À ces périodes où se mettent en place, à partir d'une « culture ancestrale : Utuleve I », des « différenciations culturelles régionales marquées par une diversification des types céramiques et la perte des décors Lapita : Utuleve II », succède une étape "dite de formation des systèmes de chefferie à titre : Utuleve II ». Aux alentours de l'an mille de notre ère, apparaît dans les marges d'un empire tongien en formation la "période d'Atulavu » : nom d'un site contemporain du règne du chef (hau) mythique Puhi. À celle-ci succède la « période des forts », vers 1400, « avec l'apparition de structures fortifiées tongiennes », l'abandon de la céramique et 